\title{
The History of Septin Biology and Bacterial Infection
}

Stevens Robertin 1 , Serge Mostowy ${ }_{1}^{*}$

1Department of Infection Biology, Faculty of Infectious and Tropical Diseases, London School of Hygiene \& Tropical Medicine, London WC1E 7HT, UK

*Author for correspondence: serge.mostowy@lshtm.ac.uk

Running head: Septins and bacterial infection

KEY WORDS: actin, autophagy, cytoskeleton, Listeria, septins, Shigella

\section{ABSTRACT}

Investigation of cytoskeleton during bacterial infection has significantly contributed to both cell and infection biology. Bacterial pathogens Listeria monocytogenes and Shigella flexneri are widely recognized as paradigms for investigation of the cytoskeleton during bacterial entry, actin-based motility and cell-autonomous immunity. At the turn of the century, septins were a poorly understood component of the cytoskeleton mostly studied in the context of yeast cell division and human cancer. In 2002, a screen performed in the laboratory of Pascale Cossart identified septin family member MSF (MLL septin-like fusion, now called SEPT9) associated with L. monocytogenes entry into human epithelial cells. These findings inspired the investigation of septins during $L$. monocytogenes and S. flexneri infection at the Institut Pasteur, illuminating important roles for septins in host-microbe interactions. In this review, we revisit the history of septin biology and bacterial infection, and discuss how the comparative study of $L$. monocytogenes and $S$. flexneri has been instrumental to understand septin roles in cellular homeostasis and host defense. 


\section{INTRODUCTION}

\section{Bacterial interactions with the actin cytoskeleton}

Rearrangements of the host cell cytoskeleton during infection are well known to promote events that benefit a bacterial pathogen, including internalization into the host cell and dissemination from cell-to-cell (Haglund and Welch, 2011). Over the past $\sim 30$ years, Listeria monocytogenes has emerged as a model bacterial pathogen used to study cytoskeleton rearrangements during infection, providing valuable insights into fundamental aspects of host cell biology (Radoshevich and Cossart, 2018). For example, L. monocytogenes has been critical to reveal a role for clathrin in bacterial invasion (Veiga and Cossart, 2005), and a role for the actin-related-protein 2/3 (ARP2/3) complex in actin-based motility (Welch et al., 1997).

Invasive bacteria promote their internalization into normally non-phagocytic host cells using two different mechanisms referred to as "zippering" and "triggering" (Cossart and Sansonetti, 2004). Zippering bacteria (such as L. monocytogenes) present invasion molecules that mimic endogenous ligands to host cell surface receptors, initiating signaling cascades that result in actin polymerization and membrane extensions which zipper around invading bacteria. Triggering bacteria (such as S. flexneri) rely on their type III secretion system (T3SS) to deliver effector proteins into the host cell cytosol that trigger actin polymerization and membrane ruffling, engulfing invading bacteria in a process similar to macropinocytosis.

Following invasion, bacterial pathogens are enclosed within an internalization vacuole (called the phagosome). In some cases, bacteria such as L. monocytogenes and S. flexneri escape to the host cell cytosol where they replicate and initiate actin-based motility by polymerization of actin at one pole of the bacterium (Welch \& Way, 2013). In this way, actin-based motility enables cytosolic bacterial pathogens to escape cell-autonomous immunity and disseminate from cell-to-cell. 


\section{The septin cytoskeleton}

As compared to actin, relatively little was known about the role of septins during bacterial infection. Septins were discovered by Nobel Laureate Leeland Hartwell while screening for cell division cycle (cdc) mutants in the budding yeast Saccharomyces cerevisiae (Hartwell, 1971). Septins have since been identified in all eukaryotes (except higher plants) and are considered a component of the cytoskeleton interacting with actin, microtubules and cellular membrane (Mostowy and Cossart, 2012). In humans, septins are classified into 4 groups (namely the SEPT2, SEPT3, SEPT6 and SEPT7 groups) based on sequence homology. Septin subunits from different groups interact through their $\mathrm{G}$ (consisting of the GTP-binding domain) and NC (consisting of the amino and carboxy-terminal regions) interfaces, forming non-polar complexes and filaments. Septin filaments can bundle and by interacting with cellular membrane form higher-order structures, such as rings.

Investigations on septin molecular functions have highlighted their roles in numerous biological processes, including cell division and motility, by acting as scaffolds for protein recruitment and diffusion barriers for subcellular compartmentalization (Bridges and Gladfelter, 2015). Recent studies have illuminated septin roles in the organization of actin and microtubule networks (Spiliotis, 2018). In agreement with septins being key for cellular homeostasis, septin dysfunction has been implicated in a wide variety of pathological conditions including cancer, male infertility and neurodegenerative disorders (Dolat, Hu, \& Spiliotis, 2014). A role for septins in bacterial infection was first suggested from studying $L$. monocytogenes invasion 20 years ago in the laboratory of Pascale Cossart (Pizarro-Cerdá et al., 2002). Since then, septins have been associated with a wide variety of bacterial pathogens, including Chlamydia trachomatis, Clostridium difficile, Salmonella Typhimurium and enteropathogenic Escherichia coli (Volceanov et al., 2014; Nölke et al., 2016; Lee et al., 2017; Boddy et al., 2018), and play important roles in host cell infection (Torraca and Mostowy, 2016; Van Ngo and Mostowy, 2019) (Figure 1). In this review, we discuss how the study of $L$. 
monocytogenes and $S$. flexneri at the Institut Pasteur launched septins into the field of bacterial infection.

\section{SEPTINS AND BACTERIAL ENTRY}

\section{Actin rearrangements during bacterial entry}

How L. monocytogenes can enter non-phagocytic cells has been investigated in great detail (Cossart and Sansonetti, 2004; Radoshevich and Cossart, 2018). Several surface proteins contribute to this process, including Internalin $(\operatorname{In} \mid \mathrm{A})$ which promotes bacterial entry through interaction with human E-cadherin, a transmembrane glycoprotein located at adherens junctions and interacting with actin through catenins (Bonazzi et al., 2009; Gaillard et al., 1991). A second L. monocytogenes invasion protein is Internalin B (InIB) which interacts with hepatocyte growth factor receptor (HGF-R / Met), a transmembrane receptor tyrosine kinase (Shen et al., 2000). Both the InIA- and InIB-mediated entry pathways require activation of the ARP2/3 complex, inducing localized polymerization of actin to promote bacterial engulfment.

\section{Septin roles in bacterial entry: from recruitment to function}

To identify new players in L. monocytogenes entry, the laboratory of Pascale Cossart used a proteomic approach based on isolation of internalization vacuoles containing InIA- or InIBcoated latex beads (Pizarro-Cerdá et al., 2002). From this, it was discovered that septin family member MSF (MLL septin-like fusion), now referred to as SEPT9 (Macara et al., 2002), is enriched at the site of InIB-mediated entry. Subsequent experiments using fluorescent microscopy showed that SEPT9 is recruited to the entry site of InIB-coated beads, where it co-localizes with actin. These observations suggested that SEPT9 regulates the InIBmediated entry of L. monocytogenes. Consistent with this, studies using bacterial cells 
revealed that SEPT9, and its interacting partners SEPT2 and SEPT11, are recruited as 0.6 $\mu \mathrm{m}$ collar-like structures with actin at the entry site of L. monocytogenes (Mostowy, Tham, et al., 2009b) (Figure 2 L1). Septin collars were similarly detected at the entry site of triggering bacteria, including $S$. flexneri (Figure 2 S1). Considering that septins function as heteroligomers and the position of SEPT9 in the human septin complex was not yet clear, studies initially focused on SEPT2 because it was viewed as central for septin complex formation and function in human cells (Sirajuddin et al., 2007). The depletion of SEPT2 using small interfering RNA (siRNA) significantly decreased the entry of $L$. monocytogenes and $S$. flexneri, revealing that SEPT2 can promote bacterial entry. Surprisingly, a separate study showed that SEPT11 restricts InIB-mediated entry by $L$. monocytogenes, highlighting that different septins may have different roles during bacterial entry (Mostowy et al., 2009a).

Importantly, treatment of cells with cytochalasin D (an inhibitor of actin polymerization) impaired septin recruitment to the site of bacterial entry, indicating that actin polymerization precedes septin assembly. Using fluorescence resonance energy transfer (FRET), it was shown that SEPT2 can activate PI 3-kinase signaling during InIB-Met interactions (Mostowy et al., 2009a). Using atomic force microscopy (AFM), it was shown that septins anchor Met to the cortical actin cytoskeleton, where it may regulate surface receptor dynamics (Mostowy et al., 2011a). At the plasma membrane, septins may act as a diffusion barrier to restrict the localization of phosphatidylinositol 4,5-bisphosphate (PI(4,5)P2)-enriched microdomains crucial for signalling processes (Sharma et al., 2013). Recent work using Salmonella Typhimurium showed that septins are involved in membrane ruffle morphology and signalling through the actin nucleating protein FHOD1 (Boddy et al., 2018). Taken together, the septin cytoskeleton can be viewed as a molecular platform modulating cytoskeletal dynamics and signal transduction events during bacterial entry. These conclusions are in agreement with work showing that septins assemble into collar-like structures at the base of the phagocytic cup during FcyR-mediated phagocytosis in macrophages and neutrophils (Huang et al., 2008). 


\section{SEPTINS AND ACTIN-BASED MOTILITY}

\section{The paradigm of the bacterial actin tail}

The observation that septins form collar-like structures around invading bacteria initiated great interest in the role of septins during other actin-based strategies exploited by intracellular pathogens, such as actin-based motility. Intracellular bacterial movement was first observed in the 1950s in cells infected with Rickettsia rickettsia, the causative agent of Rocky Mountain spotted fever (Schaechter et al., 1957), and direct evidence for bacterial actin-based motility came in the 1980s from cells infected with L. monocytogenes (Tilney and Portnoy, 1989) and S. flexneri (Bernardini et al., 1989). In the case of $L$. monocytogenes, actin tail formation requires expression of the surface protein ActA at the bacterial cell pole, which acts as a mimic of the nucleation promoting factor Wiskott-Aldrich syndrome protein (WASP) to recruit and activate the ARP2/3 complex (Welch et al., 1997). In contrast, S. flexneri expresses the bacterial autotransporter protein IcsA (also called VirG), whose activity recruits the neural Wiskott-Aldrich syndrome protein (N-WASP) and consequently ARP2/3 to the bacterial cell pole (Bernardini et al., 1989; Makino et al., 1986). Polar localization of IcsA is essential for actin tail formation, and a recent study showed that polymerization of MreB (the bacterial actin homologue) promotes polar IcsA positioning for actin tail formation (Krokowski et al., 2019).

Actin-based motility is used by various pathogens for dissemination within and between cells (Gouin, Welch, \& Cossart, 2005; Welch \& Way, 2013). However, infected host cells have powerful mechanisms to restrict actin-based motility, highlighting unforeseen aspects of cellautonomous immunity.

\section{Enter the Shigella septin cage}


Consistent with studies showing that septins are recruited to actin rich sites of bacterial entry, septins form ring-like structures around approximately $25 \%$ of L. monocytogenes and $S$. flexneri actin tails (Mostowy et al., 2010) (Figure 2 L2 and S2). The depletion of SEPT2 or SEPT9 had no significant impact on bacterial directionality or movement, indicating that septin recruitment is not essential for actin-based motility. In agreement with this, septins were not detected among proteins identified by mass-spectrometry of $L$. monocytogenes actin tails (Van Troys et al., 2008). Importantly, L. monocytogenes $\Delta a c t A$ and S. flexneri $\Delta i c s A$, isogenic mutants unable to polymerize actin tails, both fail to recruit septin (Mostowy et al., 2010). Together, these experiments suggested that septin rings assemble at sites of actin polymerization and depend on actin polymerization.

Strikingly, cytosolic $S$. flexneri surrounded by an actin cloud, though without an actin tail, are surrounded by septin cage-like structures (Figure 2 S5). In this case, septin cage-like structures compartmentalize approximately $15-30 \%$ of cytosolic S. flexneri (Mostowy et al., 2010). SEPT2 or SEPT9 depletion significantly increased the number of $S$. flexneri with actin tails, indicating that septins restrict actin tail formation. Similarly, inhibiting myosin II function using siRNA or blebbistatin increased the number of $S$. flexneri with actin tails. Treatment of cells with Tumour Necrosis Factor a (TNFa), a pleiotropic cytokine shown to stimulate association of myosin II to SEPT2, promotes septin caging and restricts actin tail formation (Mostowy et al., 2010). These results highlight septin caging as a host defense mechanism counteracting actin-based motility to restrict the dissemination of $S$. flexneri. Since its discovery in S. flexneri-infected tissue culture cells, septin cage-like structures have been observed in vivo in S. flexneri-infected zebrafish larvae (Mostowy et al., 2013), and have also been observed in tissue culture cells infected with Mycobacterium marinum (Mostowy et al., 2010), enteropathogenic Escherichia coli (EPEC) (Lee et al., 2017) and vaccinia virus (Pfanzelter et al., 2018). In the case of vaccinia virus, septins exert their antiviral effect by forming rings around virions at the plasma membrane to suppress their release from infected cells. 
Discovery of the $S$. flexneri septin cage revealed a new role for the cytoskeleton in cellautonomous immunity by inhibiting actin-based motility. Since then, another mechanism inhibiting actin-based motility has been described: the coating of cytosolic $S$. flexneri by the interferon-inducible GTPase family of guanylate-binding proteins (GBPs) (Li et al., 2017; Wandel et al., 2017; Piro et al., 2017). In this case, S. flexneri antagonizes GBP-mediated cellular defenses by secreting the E3 ubiquitin ligase IpaH9.8, which ubiquitinates and degrades GBPs in a proteasome-dependent manner. In the future, it will be of great interest to explore the relationship between septin cages and GBP coats.

Why do only a fraction of cytosolic S. flexneri recruit septins? Considering that bacterial pathogens often manipulate host cell proteins and processes to evade cell-autonomous immunity (Huang and Brumell, 2014), it is highly likely that S. flexneri has evolved mechanisms to evade septin cage entrapment. For example, post-translational modification of host cell proteins by bacterial pathogens is well known to interfere with protein activity (Salomon and Orth, 2013). Septins are regulated by a variety of post-translational modifications including acetylation, phosphorylation, ubiquitination and sumoylation (Koch et al., 2015). New work has shown that the $S$. flexneri effector IcsB is an 18-carbon fatty acyltransferase catalysing the lysine $\mathrm{N}_{\varepsilon}$-fatty acylation of SEPT7 and SEPT11 (Liu et al., 2018). Whether posttranslational modifications may affect septin cage assembly, and whether bacteria can interfere with this process, remains to be determined.

\section{OTHER SEPTIN ROLES IN CELL-AUTONOMOUS IMMUNITY}

\section{Septins and autophagy}

Autophagy delivers cytosolic material enclosed in a double-membraned vacuole (called the autophagosome) to the lysosome for degradation (Levine et al., 2011). In this way, autophagy acts as a cytosolic quality control mechanism, eliminating protein aggregates, damaged 
organelles and intracellular microbes to maintain cellular homeostasis. Targeting of bacteria to autophagy can proceed through ubiquitin-dependent and -independent signaling pathways (Khaminets et al., 2016). Ubiquitinated bacteria are recognized by autophagy receptors such as p62 (sequestosome 1 or SQSTM1) and NDP52 (nuclear dot protein, $52 \mathrm{kDa}$ ), pattern recognition receptors called sequestosome 1/p62-like receptors (SLRs) that recruit membranes for autophagosome formation through their interaction with ATG8 family proteins such as LC3B (Johansen and Lamark, 2019). Targeting of bacteria can also proceed through ubiquitin-independent pathways, such as LC3-associated phagocytosis (LAP) or opsonization by complement (Mostowy, 2013; Sorbara et al., 2018).

However, many intracellular bacteria have mechanisms to avoid recognition by autophagy (Huang \& Brumell, 2014). In the case of L. monocytogenes, avoidance of autophagy is mediated via ActA by promoting recruitment of actin-polymerization machinery on the bacterial surface, and also by enabling bacteria to move away from autophagic membranes via actinbased motility (Cheng et al., 2018; Yoshikawa et al., 2009) (Figure 2 L3). In addition to ActA, L. monocytogenes phospholipase C (PLC) can reduce the intracellular levels of phosphatidylinositol 3-phosphate (PtdIns3P), a lipid required for LC3B lipidation (Tattoli et al., 2013; Mitchell et al., 2015). In the case of S. flexneri, avoidance of autophagy is mediated via the bacterial effector protein IcsB, which prevents the recruitment of ATG5 (a protein critical for autophagosome maturation) to IcsA (Ogawa et al., 2005). A more recent study discovered that $\mathrm{N}_{\varepsilon}$-fatty acyltransferase activity of IcsB modifies CHMP5 (a component of the ESCRT-III complex) to escape from autophagy (Liu et al., 2018). In addition to IcsB, S. flexneri VirA can inactivate Rab1 to counteract autophagy (Dong et al., 2012).

Several autophagy markers (e.g., p62, NDP52 and LC3B) are recruited to septin cages (Mostowy et al., 2010; Mostowy et al., 2011b) (Figure 2 S6), and S. flexneri IcsB mutants are compartmentalized in septin cages more efficiently than the wild-type strain (Mostowy et al., 2010), consistent with a role for IcsB in autophagy escape (Ogawa et al., 2005; Liu et al., 
2018). Strikingly, when septins (SEPT2 or SEPT9) or autophagy components (p62, ATG5, ATG6 or ATG7) are depleted by siRNA, both septin cages and autophagy markers fail to recruit around S. flexneri (Mostowy et al., 2010). These results highlight an interdependence between septin assembly and autophagy. In agreement with this, a study using proteomics found autophagy and mitochondrial proteins associated with septins in $S$. flexneri-infected cells (Figure 2 S4), and that actin-polymerizing S. flexneri fragment mitochondria to escape from septin cage-mediated autophagy (Sirianni et al., 2016).

In a first study to investigate bacterial autophagy in vivo, infection of zebrafish larvae with $S$. flexneri highlighted autophagy as a crucial component of innate immunity at the whole animal level (Mostowy et al., 2013). Here, depletion of p62 using morpholino oligonucleotide significantly reduced the number of septin cage-associated $S$. flexneri, and significantly increased bacterial burden. In agreement with the view that septin cages are anti-bacterial, work using HeLa cells showed that approximately $50 \%$ of $S$. flexneri entrapped in septin cages are not metabolically active (Sirianni et al., 2016). On the other hand, septins and the autophagy machinery can regulate metabolic pathways that promote the proliferation of intracellular S. flexneri not entrapped in septin cages (Lobato-Márquez et al., 2019).

Although the fate of $S$. flexneri in septin cages is relatively well characterized, how septins sense cytosolic bacteria was mostly unknown. It has been recognized for approximately 10 years that septin assembly is membrane facilitated (Tanaka-Takiguchi et al., 2009), and recent work suggested that septins sense micron-scale curvature at the plasma membrane (Bridges et al., 2016). In agreement with this, a recent study showed that septins are recruited to regions of micron-scale curvature presented by dividing bacterial cells (Figure 2 S3), and cardiolipin (a curvature-specific phospholipid) promotes septin recruitment to these regions (Krokowski et al., 2018). Following pharmacological inhibition of bacterial cell division, septins are recruited to bacterial cell poles but fail to assemble into cages, indicating that bacterial cell growth is required for septin cage entrapment. Therefore, bacterial cell division can be viewed 
as a fundamental danger signal used by the host cell to recognize intracellular bacteria (Krokowski et al., 2018; Van Ngo and Mostowy, 2019; Krokowski and Mostowy, 2019).

\section{Septins and inflammation}

A growing number of studies have indicated that the cytoskeleton plays key roles during inflammation (Mostowy and Shenoy, 2015). For example, actin has been linked to inflammation control through regulation of the NACHT, LRR and PYD domain-containing protein 3 (NLRP3) inflammasome, a large multimeric complex that activate caspase- 1 and pro-inflammatory cytokines (Burger et al., 2016). Following cellular damage or infection, inflammasome formation and pyroptosis (an inflammatory type of programmed cell death) is linked with disruption of actin and microtubules (Davis et al., 2019). Although a role for septins in inflammasome activity has not yet been shown, recent studies have linked septins to dysregulated inflammatory responses (Lee et al., 2017; Mazon-Moya et al., 2017). In the case of human monocytic THP-1 cells infected with S. flexneri, disruption of WASP by short hairpin RNA (shRNA) reduced septin caging and increased inflammasome activity and host cell death

(Lee et al., 2017). At the whole animal level, work using the S. flexneri-zebrafish infection model discovered an in vivo role for septins in inflammation control (Mazon-Moya et al., 2017). Here, depletion of Sept7b or Sept15 (zebrafish orthologues of human SEPT7) using morpholino oligonucleotide significantly increased host susceptibility to $S$. flexneri infection. Moreover, Sept15 morphants exhibit significantly increased caspase-1 activity and host cell death, indicating that septins restrict inflammation in vivo. Taken together, these results suggest that septins may interact with the inflammasome, and factors important in septinmediated restriction of inflammation can represent novel drug targets for the control of bacterial infection and inflammatory disorders. 


\section{CONCLUSION AND FUTURE PERSPECTIVES}

The study of infection by L. monocytogenes and S. flexneri has significantly advanced our understanding of cellular microbiology and cell-autonomous immunity. In vitro reconstitution of L. monocytogenes and S. flexneri actin-based motility has been essential to identify key proteins underlying actin polymerization (Loisel et al.,, 1999;Welch et al., 1998). Similarly, in vitro reconstitution of the $S$. flexneri septin cage may yield important insights on the biochemical, molecular and structural aspects of septin-microbe interactions. To elucidate the precise molecular basis of $S$. flexneri septin caging, the use of high resolution (such as cryoelectron tomography) and high content (to screen host and/or bacterial factors) microscopy techniques will be of great interest. Although this review is focused on L. monocytogenes and S. flexneri infection, it is essential to understand the full breadth of microbial pathogens regulated by septin biology, and to test how widespread is the role of cell shape and membrane curvature in pathogen sensing (Torraca and Mostowy, 2016; Van Ngo and Mostowy, 2019). Our current knowledge of septin function during infection mostly derives from work performed in vitro using non-phagocytic cells (e.g. HeLa); future work should address septin function in immune cells (e.g. macrophages, neutrophils) and whole animal models (e.g. zebrafish and mice). Overall, the study of septin biology and bacterial infection has provided unexpected insights into septin biology, and septin recruitment by a wide range of pathogens will likely continue to highlight novel septin functions. This information may help to elucidate therapeutic treatments for infection, inflammation and other human diseases in which septins have been implicated.

\section{ACKNOWLEDGEMENTS}

We apologize to authors whose work we could not cite due to space limitations. Work in the SM laboratory is supported by a European Research Council Consolidator Grant (772853 ENTRAPMENT), Wellcome Trust Senior Research Fellowship (206444/Z/17/Z) and the Lister Institute of Preventive Medicine. 


\section{CONFLICT OF INTEREST}

The authors declare no conflict of interest.

\section{REFERENCES}

Bai, X., Bowen, J.R., Knox, T.K., Zhou, K., Pendziwiat, M., Kuhlenbäumer, G., et al. (2013) Novel septin 9 repeat motifs altered in neuralgic amyotrophy bind and bundle microtubules. J Cell Biol 203: 895-905.

Bernardini, M.L., Mounier, J., D'Hauteville, H., Coquis-Rondon, M., and Sansonetti, P.J. (1989) Identification of icsA, a plasmid locus of Shigella flexneri that governs bacterial intraand intercellular spread through interaction with F-actin. Proc Natl Acad Sci U S A 86: 38673871.

Boddy, K.C., Gao, A.D., Truong, D., Kim, M.S., Froese, C.D., Trimble, W.S., and Brumell, J.H. (2018) Septin-regulated actin dynamics promote Salmonella invasion of host cells. Cell Microbiol 20: 1-10.

Bonazzi, M., Lecuit, M., and Cossart P. (2009) Listeria monocytogenes internalin and Ecadherin: from bench to bedside. Cold Spring Harb. Perspect. Biol. 1:a003087. 10.1101/cshperspect.a003087,

Bridges, A.A., and Gladfelter, A.S. (2015) Septin form and function at the cell cortex. J Biol Chem 290: 17173-17180.

Bridges, A.A., Jentzsch, M.S., Oakes, P.W., Occhipinti, P., and Gladfelter, A.S. (2016) Micronscale plasma membrane curvature is recognized by the septin cytoskeleton. J Cell Bio/ 213: 23-32 http://jcb.rupress.org/content/213/1/23.

Burger, D., Fickentscher, C., Moerloose, P. De, and Brandt, K.J. (2016) F-actin dampens NLRP3 inflammasome activity via Flightless-I and LRRFIP2. Sci Rep 6: 1-12 http://dx.doi.org/10.1038/srep29834.

Cheng, M.I., Chen, C., Engström, P., Portnoy, D.A., and Mitchell, G. (2018) Actin-based 
motility allows Listeria monocytogenes to avoid autophagy in the macrophage cytosol. Cell Microbiol 20: e12854.

Cossart, P., and Sansonetti, P.J. (2004) Bacterial Invasion: The paradigms of enteroinvasive pathogens. Science (80- ) 304: 242-248.

Davis, M.A., Fairgrieve, M.R., Hartigh, A. Den, Yakovenko, O., Duvvuri, B., Lood, C., et al. (2019) Calpain drives pyroptotic vimentin cleavage, intermediate filament loss, and cell rupture that mediates immunostimulation. Proc Natl Acad Sci 116: 5061 LP -5070 http://www.pnas.org/content/116/11/5061.abstract.

Dolat, L., Hu, Q., and Spiliotis, E.T. (2014) Septin functions in organ system physiology and pathology. Biol Chem 395: 123-141 https://www.ncbi.nlm.nih.gov/pubmed/24114910.

Dong, N., Zhu, Y., Lu, Q., Hu, L., Zheng, Y., and Shao, F. (2012) Structurally distinct bacterial TBC-like GAPs link Arf GTPase to Rab1 inactivation to counteract host defenses. Cell 150: 1029-1041 http://dx.doi.org/10.1016/j.cell.2012.06.050.

Gaillard, J.-L., Berche, P., Frehel, C., Gouln, E., and Cossart, P. (1991) Entry of L. monocytogenes into cells is mediated by internalin, a repeat protein reminiscent of surface $\begin{array}{lllll}\text { antigens from } & \text { gram-positive } & \text { cocci. } & \text { Cell } & \text { 65: }\end{array}$ http://www.sciencedirect.com/science/article/pii/009286749190009N.

Gouin, E., Welch, M.D., and Cossart, P. (2005) Actin-based motility of intracellular pathogens. $\begin{array}{lllll}\text { Curr } & \text { Opin } & \text { Microbiol } & \text { 8: } & \text { 35-45 }\end{array}$ http://www.sciencedirect.com/science/article/pii/S1369527404001675.

Haglund, C.M., and Welch, M.D. (2011) Pathogens and polymers: Microbe-host interactions illuminate the cytoskeleton. J Cell Biol 195: 7-17.

Hartwell, L.H. (1971) Genetic control of the cell division cycle in yeast. IV. Genes controlling bud emergence and cytokinesis. Exp Cell Res 69: 265-276.

Huang, J., and Brumell, J.H. (2014b) Bacteria-autophagy interplay: A battle for survival. Nat Rev Microbiol 12: 101-114 http://dx.doi.org/10.1038/nrmicro3160.

Huang, Y.-W., Yan, M., Collins, R.F., Diciccio, J.E., Grinstein, S., and Trimble, W.S. (2008) Mammalian septins are required for phagosome formation. Mol Biol Cell 19: 1717-1726. 
Johansen, T., and Lamark, T. (2019) Selective Autophagy: ATG8 Family Proteins, LIR Motifs and Cargo Receptors. J Mol Biol https://doi.org/10.1016/j.jmb.2019.07.016.

Kayath, C.A., Hussey, S., hajjami, N. El, Nagra, K., Philpott, D., and Allaoui, A. (2010) Escape of intracellular Shigella from autophagy requires binding to cholesterol through the type III $\begin{array}{llll}\text { effector, } & \text { IcsB. } & \text { Microbes } & \text { Infect }\end{array}$ http://www.sciencedirect.com/science/article/pii/S1286457910001589.

Khaminets, A., Behl, C., and Dikic, I. (2016) Ubiquitin-dependent and independent signals in $\begin{array}{lllll}\text { selective } & \text { autophagy. } & \text { Trends } & \text { 6ell }\end{array}$ http://www.sciencedirect.com/science/article/pii/S0962892415001610.

Koch, S., Acebron, S.P., Herbst, J., Hatiboglu, G., and Niehrs, C. (2015) Post-transcriptional Wnt signaling governs epididymal sperm maturation. Cell 163: 1225-1236 http://www.sciencedirect.com/science/article/pii/S0092867415013434.

Kocks, C., Gouin, E., Tabouret, M., Berche, P., Ohayon, H., and Cossart, P. (1992) L. monocytogenes-induced actin assembly requires the actA gene product, a surface protein. Cell 68: 521-531.

Krokowski, S., Atwal, S., Lobato-Márquez, D., Chastanet, A., Carballido-López, R., Salje, J., and Mostowy, S. (2019) Shigella MreB promotes polar IcsA positioning for actin tail formation. J Cell Sci 132: 1-9.

Krokowski, S., Lobato-Márquez, D., Chastanet, A., Pereira, P.M., Angelis, D., Galea, D., et al. (2018) Septins recognize and entrap dividing bacterial cells for delivery to lysosomes. Cell Host Microbe 24: 866-874.e4.

Krokowski, S., and Mostowy, S. (2019) Bacterial cell division is recognized by the septin cytoskeleton for restriction by autophagy. Autophagy 15: 937-939.

Lee, P.P., Lobato-Márquez, D., Pramanik, N., Sirianni, A., Daza-Cajigal, V., Rivers, E., et al. (2017) Wiskott-Aldrich syndrome protein regulates autophagy and inflammasome activity in innate immune cells. Nat Commun 8: 1576 https://doi.org/10.1038/s41467-017-01676-0.

Li, P., Jiang, W., Yu, Q., Liu, W., Zhou, P., Li, J., et al. (2017) Ubiquitination and degradation of GBPs by a Shigella effector to suppress host defence. Nature 551: 378-383. 
Liu, W., Zhou, Y., Peng, T., Zhou, P., Ding, X., Li, Z., et al. (2018) N(ع)-fatty acylation of multiple membrane-associated proteins by Shigella IcsB effector to modulate host function. Nat Microbio/ 3: 996-1009.

Lobato-Márquez, D., Krokowski, S., Sirianni, A., Larrouy-Maumus, G., and Mostowy, S. (2019) A requirement for septins and the autophagy receptor p62 in the proliferation of intracellular Shigella. Cytoskeleton (Hoboken) 76: 163-172.

Loisel, T.P., Boujemaa, R., Pantaloni, D., and Carlier, M.-F. (1999) Reconstitution of actinbased motility of Listeria and Shigella using pure proteins. Nature 401: 613-616 https://doi.org/10.1038/44183.

Macara, I.G., Baldarelli, R., Field, C.M., Glotzer, M., Hayashi, Y., Hsu, S.-C., et al. (2002) Mammalian septins nomenclature. Mol Biol Cell 13: 4111-4113 https://doi.org/10.1091/mbc.e02-07-0438.

Makino, S., Sasakawa, C., Kamata, K., Kurata, T., and Yoshikawa, M. (1986) A genetic determinant required for continuous reinfection of adjacent cells on large plasmid in S. flexneri 2a. Cell 46: 551-555.

Mazon-Moya, M.J., Willis, A.R., Torraca, V., Boucontet, L., Shenoy, A.R., Colucci-Guyon, E., and Mostowy, S. (2017) Septins restrict inflammation and protect zebrafish larvae from Shigella infection. PLOS Pathog 13: 1-22 https://doi.org/10.1371/journal.ppat.1006467.

Mitchell, G., Ge, L., Huang, Q., Chen, C., Kianian, S., Roberts, M.F., et al. (2015) Avoidance of autophagy mediated by PICA or ActA is required for Listeria monocytogenes growth in macrophages. Infect Immun 83: 2175-2184.

Mostowy, S. (2013) Autophagy and bacterial clearance: A not so clear picture. Cell Microbiol 15: 395-402.

Mostowy, S., Bonazzi, M., Hamon, M.A., Tham, T.N., Mallet, A., Lelek, M., et al. (2010) Entrapment of intracytosolic bacteria by septin cage-like structures. Cell Host Microbe 8: 433444.

Mostowy, S., Boucontet, L., Mazon Moya, M.J., Sirianni, A., Boudinot, P., Hollinshead, M., et al. (2013) The zebrafish as a new model for the in vivo study of Shigella flexneri interaction 
with phagocytes and bacterial autophagy. PLoS Pathog 9: 12-16.

Mostowy, S., and Cossart, P. (2012) Septins: The fourth component of the cytoskeleton. Nat Rev Mol Cell Biol 13: 183-194 http://dx.doi.org/10.1038/nrm3284.

Mostowy, S., Danckaert, A., Tham, T.N., Machu, C., Guadagnini, S., Pizarro-Cerdá, J., and Cossart, P. (2009a) Septin 11 restricts InIB-mediated invasion by Listeria. J Biol Chem 284: $11613-11621$.

Mostowy, S., Janel, S., Forestier, C., Roduit, C., Kasas, S., Pizarro-Cerdá, J., et al. (2011a) A role for septins in the interaction between the Listeria monocytogenes invasion protein InIB and the Met receptor. Biophys J 100: 1949-1959.

Mostowy, S., Sancho-Shimizu, V., Hamon, M.A., Simeone, R., Brosch, R., Johansen, T., and Cossart, P. (2011b) p62 and NDP52 proteins target intracytosolic Shigella and Listeria to different autophagy pathways. J Biol Chem 286: 26987-26995.

Mostowy, S., and Shenoy, A.R. (2015) The cytoskeleton in cell-autonomous immunity: Structural determinants of host defence. Nat Rev Immunol 15: 559-573 http://dx.doi.org/10.1038/nri3877.

Mostowy, S., Tham, T.N., Danckaert, A., Guadagnini, S., Boisson-Dupuis, S., Pizarro-Cerdá, J., and Cossart, P. (2009b) Septins regulate bacterial entry into host cells. PLoS One 4. Ngo, H. Van, and Mostowy, S. (2019) Role of septins in microbial infection. J Cell Sci 132. Nölke, T., Schwan, C., Lehmann, F., Østevold, K., Pertz, O., and Aktories, K. (2016) Septins guide microtubule protrusions induced by actin-depolymerizing toxins like Clostridium difficile transferase (CDT). Proc Natl Acad Sci U S A 113: 7870-7875.

Ogawa, M., Yoshimori, T., Suzuki, T., Sagara, H., Mizushima, N., and Sasakawa, C. (2005a) Escape of intracellular Shigella from autophagy. Science (80- ) 307: 727-731 https://science.sciencemag.org/content/307/5710/727.

Pfanzelter, J., Mostowy, S., and Way, M. (2018) Septins suppress the release of vaccinia virus from infected cells. J Cell Bio/ 217: 2911-2929.

Piro, A.S., Hernandez, D., Luoma, S., Feeley, E.M., Finethy, R., Yirga, A., et al. (2017) Detection of cytosolic Shigella flexneri via a C-terminal triple-arginine motif of GBP1 Inhibits 
Actin-Based Motility. MBio 8: e01979-17 http://mbio.asm.org/content/8/6/e01979-17.abstract. Pizarro-Cerdá, J., Jonquières, R., Gouin, E., Vandekerckhove, J., Garin, J., and Cossart, P. (2002) Distinct protein patterns associated with Listeria monocytogenes InIA- or InIBphagosomes. Cell Microbiol 4: 101-115.

Radoshevich, L., and Cossart, P. (2018) Listeria monocytogenes: Towards a complete picture of its physiology and pathogenesis. Nat Rev Microbiol 16: 32-46 http://dx.doi.org/10.1038/nrmicro.2017.126.

Salomon, D., and Orth, K. (2013) What pathogens have taught us about posttranslational $\begin{array}{llll}\text { modifications. } & \text { Cell } & \text { Host } & \text { 269-279 }\end{array}$ https://www.ncbi.nlm.nih.gov/pubmed/24034613.

Schaechter, M., Bozeman, F.M., and Smadel, J.E. (1957) Study on the growth of rickettsiae: II. Morphologic observations of living rickettsiae in tissue culture cells. Virology 3: 160-172 http://www.sciencedirect.com/science/article/pii/0042682257900302.

Sharma, S., Quintana, A., Findlay, G.M., Mettlen, M., Baust, B., Jain, M., et al. (2013) An siRNA screen for NFAT activation identifies septins as coordinators of store-operated Ca2+ entry. Nature 499: 238-242 https://www.ncbi.nlm.nih.gov/pubmed/23792561.

Shen, Y., Naujokas, M., Park, M., and Ireton, K. (2000) InIB-dependent internalization of Listeria is mediated by the Met receptor tyrosine kinase. Cell 103: 501-510.

Sirajuddin, M., Farkasovsky, M., Hauer, F., Kühlmann, D., Macara, I.G., Weyand, M., et al. (2007) Structural insight into filament formation by mammalian septins. Nature 449: 311-315 https://www.ncbi.nlm.nih.gov/pubmed/17637674.

Sirianni, A., Krokowski, S., Lobato-Márquez, D., Buranyi, S., Pfanzelter, J., Galea, D., et al. (2016) Mitochondria mediate septin cage assembly to promote autophagy of Shigella. EMBO Rep 17: 1029-1043.

Sorbara, M.T., Foerster, E.G., Tsalikis, J., Abdel-Nour, M., Mangiapane, J., Sirluck-Schroeder, I., et al. (2018) Complement C3 drives autophagy-dependent restriction of cyto-invasive bacteria. Cell Host Microbe 23: 644-652.e5 https://www.ncbi.nlm.nih.gov/pubmed/29746835. Spiliotis, E.T. (2018) Spatial effects-site-specific regulation of actin and microtubule 
organization by septin GTPases. J Cell Sci 131: 1-10.

Tanaka-Takiguchi, Y., Kinoshita, M., and Takiguchi, K. (2009) Septin-mediated uniform bracing of phospholipid membranes. Curr Biol 19: 140-145 http://www.sciencedirect.com/science/article/pii/S0960982208016850.

Tattoli, I., Sorbara, M.T., Yang, C., Tooze, S.A., Philpott, D.J., and Girardin, S.E. (2013) Listeria phospholipases subvert host autophagic defenses by stalling pre-autophagosomal structures. EMBO J 32: 3066-3078.

Tilney, L.G., and Portnoy, D.A. (1989) Actin filaments and the growth, movement, and spread of the intracellular bacterial parasite, Listeria monocytogenes. J Cell Biol 109: 1597-1608.

Torraca, V., and Mostowy, S. (2016) Septins and bacterial infection. Front Cell Dev Biol 4: 18.

Torraca, V., and Mostowy, S. (2018) Zebrafish Infection: From Pathogenesis to Cell Biology. Trends Cell Biol 28: 143-156 http://dx.doi.org/10.1016/j.tcb.2017.10.002.

Troys, M. Van, Lambrechts, A., David, V., Demol, H., Puype, M., Pizarro-Cerda, J., et al. (2008) The actin propulsive machinery: the proteome of Listeria monocytogenes tails. Biochem Biophys Res Commun 375: 194-199.

Veiga, E., and Cossart, P. (2005) Listeria hijacks the clathrin-dependent endocytic machinery to invade mammalian cells. Nat Cell Biol 7: 894-900.

Verdier-Pinard, P., Salaun, D., Bouguenina, H., Shimada, S., Pophillat, M., Audebert, S., et al. (2017) Septin 9-i2 is downregulated in tumors, impairs cancer cell migration and alters subnuclear actin filaments. Sci Rep 7: 1-18.

Volceanov, L., Herbst, K., Biniossek, M., Schilling, O., Haller, D., Nölke, T., et al. (2014) Septins arrange F-actin-containing fibers on the Chlamydia trachomatis inclusion and are required for normal release of the inclusion by extrusion. MBio 5: e01802-14.

Wandel, M.P., Pathe, C., Werner, E.I., Ellison, C.J., Boyle, K.B., Malsburg, A. von der, et al. (2017) GBPs inhibit motility of Shigella flexneri but are targeted for degradation by the bacterial ubiquitin ligase IpaH9.8. Cell Host Microbe 22: 507-518.e5 https://doi.org/10.1016/j.chom.2017.09.007. 
Welch, M.D., Iwamatsu, A., and Mitchison, T.J. (1997) Actin polymerization is induced by Arp2/3 protein complex at the surface of Listeria monocytogenes. Nature 385: 265-269 http://dx.doi.org/10.1038/385265a0.

Welch, M.D., Rosenblatt, J., Skoble, J., Portnoy, D.A., and Mitchison, T.J. (1998) Interaction of human Arp2/3 complex and the Listeria monocytognes ActA protein in actin filament nucleation. Science (80- ) 281: 105-108.

Welch, M.D., and Way, M. (2013) Arp2/3-mediated actin-based motility: a tail of pathogen abuse. Cell Host Microbe 14: 242-255.

Yoshikawa, Y., Ogawa, M., Hain, T., Yoshida, M., Fukumatsu, M., Kim, M., et al. (2009) Listeria monocytogenes ActA-mediated escape from autophagic recognition. Nat Cell Biol 11: $1233-1240$. 


\section{FIGURE LEGENDS}

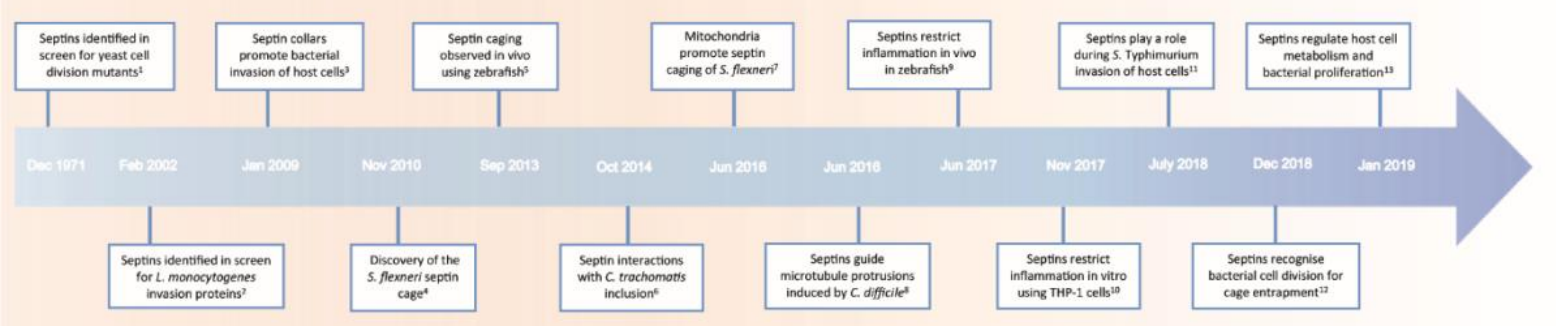

Figure 1. Timeline: A history of septin biology and bacterial infection

1) Septins were discovered using mutagenesis screens in yeast to find genes crucial for cell division (Hartwell, 1971); 2) In the case of infection biology, septins were discovered at the Institut Pasteur using a proteomic screen to find genes crucial for L. monocytogenes entry (Pizarro-Cerdá et al., 2002); 3) Septins assemble as collar-like structures around invading bacteria, launching septins into the field of bacterial infection (Mostowy et al., 2009b); 4) Septins assemble into cage-like structures around actin-polymerizing $S$. flexneri, revealing a new link between the cytoskeleton and cell-autonomous immunity (Mostowy et al., 2010); 5) Septin caging observed in vivo using $S$. flexneri infection of zebrafish, a first animal model testing the role of bacterial autophagy in vivo (Mostowy et al., 2013); 6) Septins organize filamentous actin around the $C$. trachomatis inclusion, and are required for inclusion release by extrusion (Volceanov et al., 2014); 7) Mitochondria promote septin assembly into cages that entrap S. flexneri for targeting to autophagy, a first report to highlight septin-mitochondria interactions (Sirianni et al., 2016); 8) Septins guide microtubule protrusions induced by $C$. difficile during host cell adhesion (Nölke et al., 2016); 9) Septins discovered to restrict 
inflammation in vivo and protect zebrafish larvae from S. flexneri infection (Mazon-Moya et al., 2017); 10) Septin-mediated autophagy in THP-1 cells observed to restrict inflammation in vitro (Lee et al., 2017); 11) Septins regulate actin dynamics to promote $S$. Typhimurium entry in host cells (Boddy et al., 2018); 12) Septins recognize micron-scale membrane curvature of dividing S. flexneri for delivery to lysosome, highlighting a new link between cell division and cell-autonomous immunity (Krokowski et al., 2018); 13) Septins and the autophagy machinery can promote the proliferation of intracellular S. flexneri not entrapped in septin cages (LobatoMárquez et al., 2019).

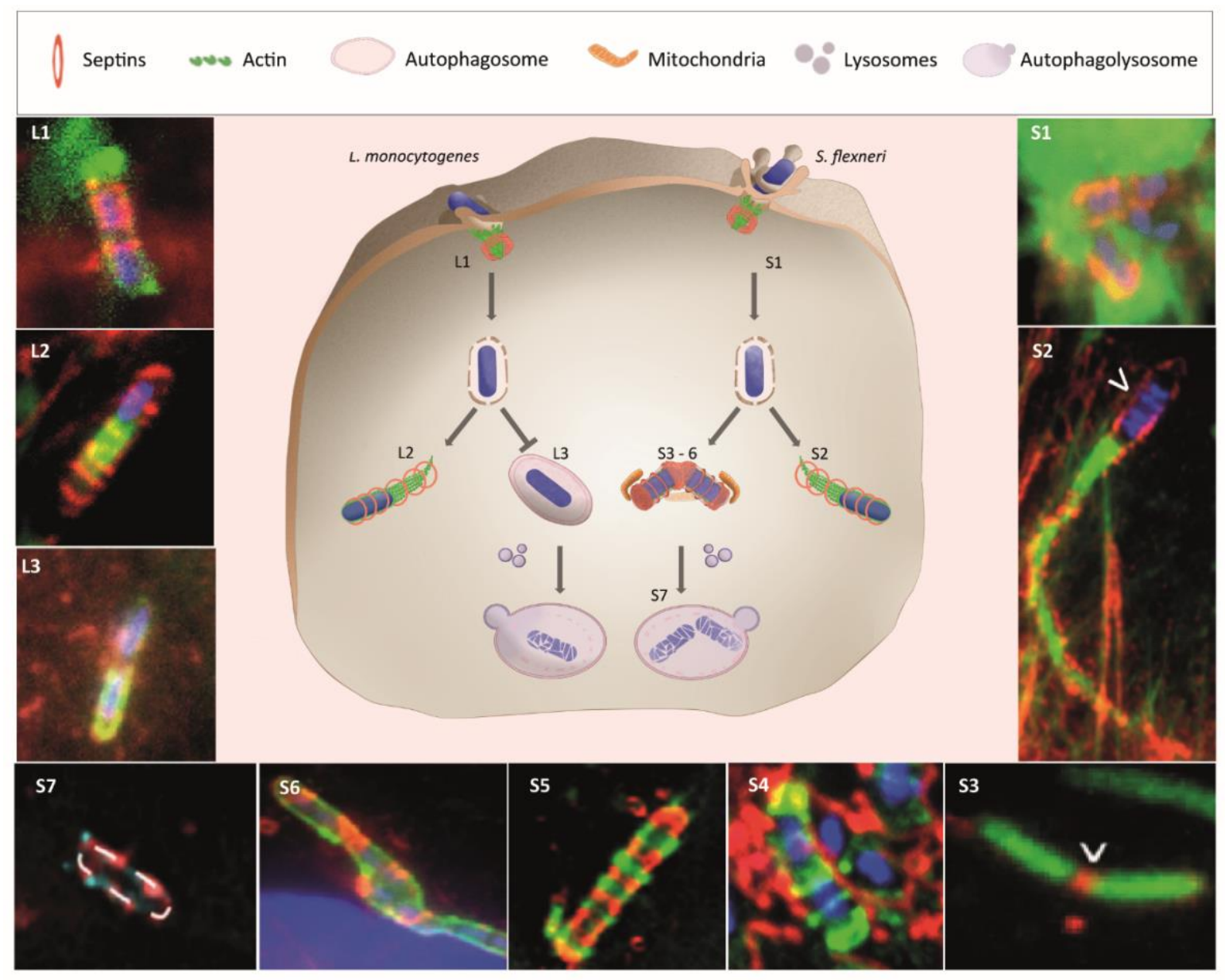

Figure 2. L. monocytogenes and S. flexneri interactions with the septin cytoskeleton (Center) Model illustrating the interaction of L. monocytogenes (left) and S. flexneri (right) with septins during infection of host cells. (Surrounding) Representative immunofluorescence 
micrographs of septin-bacteria interactions. (L1, S1) Septin recruitment to the site of $L$. monocytogenes and S. flexneri entry in Jeg-3 and HeLa cells, respectively. Endogenous septin stained with anti-SEPT9 (L. monocytogenes) or anti-SEPT2 (S. flexneri), actin stained with anti-F-actin (green), and bacteria marked using DAPI (blue). Images adapted with permission from (Mostowy et al., 2009b). (L2, S2) Septin recruitment to actin tail of $L$. monocytogenes and S. flexneri in Jeg3 cells and HeLa cells, respectively. Endogenous septin stained with anti-SEPT9, actin stained with phalloidin (green), and bacteria marked using DAPI (blue). Images adapted with permission from (Mostowy et al., 2010). (L3) Recruitment of autophagy markers to L. monocytogenes $\triangle a c t A$ in HeLa cells. p62 stained with anti-p62 (green), NDP52 stained with anti-NDP52 (red), and bacteria marked using DAPI (blue). Image adapted with permission from (Mostowy et al., 2011b). (S3) Septins are recruited to micronscale curvature of dividing intracellular bacteria. SEPT6-GFP HeLa cells were infected with $S$. flexneri mCherry. White arrowheads indicate septin recruitment to the bacterial division site. Image adapted with permission from (Krokowski et al., 2018). (S4) Mitochondria promote septin cage assembly. HeLa cells stably expressing SEPT6-GFP were transfected with MitoBFP and infected with $S$. flexneri mCherry. SEPT6 is shown in green, mitochondria in red and S. flexneri-mCherry in blue. Image adapted with permission from (Sirianni et al., 2016). (S5) The S.flexneri-septin cage. Endogenous septin stained with anti-SEPT9, actin stained with phalloidin (green), and S. flexneri marked using DAPI (blue). Image adapted with permission from (Mostowy et al., 2010). (S6) Septin caged bacteria are targeted to autophagy in HeLa cells expressing ATG8-GFP. Endogenous septin stained with anti-SEPT2 (red) and S. flexneri marked using DAPI (blue). Image adapted with permission from (Mostowy et al., 2010). (S7) Septins target bacteria to lysosomal degradation. SEPT6-GFP HeLa cells labelled with LysoTracker red. Septin cage-entrapped S. flexneri is shown as dashed white outline. Image adapted with permission from (Krokowski et al., 2018). 